\title{
Características del terreno de fundación de sitios con edificios dañados severamente en el terremoto del 27F
}

Characteristics of the foundation ground of sites with severely damaged buildings in the $27 \mathrm{~F}$ earthquake

\section{Loreto Vergara y Ramón Verdugo}

Fecha de entrega: 5 de enero 2017 Fecha de aceptación: 22 de mayo 2017

CMGI Ltda., Virginia Opazo 48, Santiago, Chile, lvergara@cmgi.cl, rverdugo@cmgi.cl

El terremoto del $27 F$ afectó a una extensa área del pais, siendo principalmente devastador en las regiones del Maule y del Bío Bío. En la Región Metropolitana se registraron importantes daños tanto en viviendas como en edificios modernos. En el caso de los edificios, las fallas variaron desde daños menores hasta irreparables. Con el objetivo de identificar si las características del terreno fue uno de los factores detonantes de los daños en edificios, se realizaron mediciones de vibraciones ambientales en las cercanias de las estructuras severamente dañadas en la Provincia de Santiago. El análisis fue realizado mediante el método de Nakamura, el cual permite obtener el periodo preponderante del terreno, especialmente en suelos de baja competencia geotécnica, los cuales presentan a su vez las mayores probabilidades de generar daños estructurales en construcciones. Se realizaron 122 mediciones ubicadas en las cercanías de 68 de los edificios más dañados en la Provincia de Santiago. Asimismo, se compararon las razones espectrales con la geología superficial del terreno, de manera de corroborar si existe una relación entre ellos. Los resultados de análisis indican que no existe relación directa entre los edificios severamente dañados y la amplificación sísmica, debido a que los edificios se emplazan sobre distintos tipos de razones espectrales $H / V$. Se concluye que el factor preponderante de los daños ocurridos estaría asociado con aspectos principalmente estructurales. Adicionalmente, la cuenca de Santiago es subdivida en tres sectores de acuerdo a los resultados obtenidos (rígido, semi-rígido y periodos predominantes sobre $0.5 \mathrm{~s}$ ).

Palabras clave: efecto de sitio, método de Nakamura, amplificación sísmica, cuenca de Santiago
The Maule Earthquake affected a wide area of the country, being significantly destructive in the regions of Maule and Bio Bio. In the Metropolitan Region major damages were reported in both housings and modern buildings. In the case of buildings, failures ranged from minor damage to unrecoverable. To identify whether the ground was one of the triggering factors that damaged the buildings, environmental vibration measurements were carried out in the vicinity of severely damaged structures in the Province of Santiago. The analysis was performed using the method of Nakamura, which may provide the predominant period of the ground, especially in soils of low geotechnical quality, which present higher probability of generating structural damage in buildings. 122 measurements located in the vicinity of 68 severely damaged buildings located in the Province of Santiago were carried out. Also, the obtained spectral ratios $H / V$ were compared with the geology of the area, in order to confirm the relationship between them. The analyses of the results indicate that there is no direct relationship between the observed damage of buildings and the seismic amplification, because the damaged buildings are located on different types of spectral ratio $H / V$. It is concluded that the main factor of the damages could be associated with structural aspects. In addition, the Santiago basin is subdivided into three sectors according to the $H / V$ spectral ratio (rigid, semi-rigid and predominant periods of $0.5 \mathrm{~s}$ ).

Keywords: site effect, Nakamura method, seismic amplification, Santiago Basin

\section{Introducción}

El mega terremoto del 27 de Febrero del 2010 se ubica entre los diez eventos sísmicos de mayor magnitud a nivel mundial, dejando un saldo de más de 2.000.000 de afectados, más de 200.000 viviendas destruidas y más de 500 víctimas fatales (Presidencia de la República, 2013). Los daños abarcaron transversalmente distintas infraestructuras, como viviendas, hospitales, blocks 
habitacionales, puentes, edificios de gran altura, etc. En la Provincia de Santiago se registraron 10705 viviendas y 560 construcciones de dimensión mayor con daño estructural (Vergara, 2014). En el caso de los edificios, se registraron desde daños locales, que permitieron el uso total o parcial del inmueble, hasta daños irreparables, que implicaron decretos de demolición.

Por otra parte, numerosos estudios han mostrado que existe una modificación de la señal sísmica dependiendo de las condiciones geotécnicas del sector, este fenómeno denominado como efecto de sitio puede resultar en una amplificación de la señal y una variación del contenido de frecuencias. El uso de vibraciones ambientales mediante el método de Nakamura es uno de los métodos más utilizados para estimar la respuesta sísmica de un depósito de suelos, el cual utiliza el cociente de los espectros horizontales y verticales medidos en superficie, permitiendo estimar la frecuencia fundamental de un depósito en particular.

Con el objetivo de identificar si el fenómeno de amplificación sísmica fue una de las causas que acrecentó el nivel de deterioro en los edificios más dañados en la Provincia de Santiago, se realizaron mediciones de vibraciones ambientales en las cercanías de éstos. Adicionalmente, se ejecutaron mediciones en las cercanías de las estaciones sismológicas ubicadas en las comunas de Maipú y Peñalolén, y en las inmediaciones del aeropuerto de Santiago, de manera de obtener una visión general de la respuesta sísmica de la cuenca de Santiago, en términos del periodo fundamental.

Adicionalmente, se comparan las razones espectrales obtenidas de mediciones, con la geología superficial y la profundidad del basamento rocoso (Vergara y Verdugo, 2014), de manera de corroborar la existencia de una relación entre el método de Nakamura y las características del sitio.

\section{Método de Nakamura 0 razones espectrales $\mathrm{H} / \mathrm{V}$}

Este método propone estimar el periodo predominante de un depósito de suelos a partir de la medición de vibraciones ambientales verticales y horizontales en superficie. Este método se originó en base al análisis de registros de movimiento en diferentes sitios de Japón, en donde se descubrió que en terrenos blandos el movimiento horizontal es mayor que el movimiento vertical, contrario a lo observado en terrenos rígidos, donde los movimientos horizontales y verticales son similares entre si (Nakamura, 2008).

El método asume que la componente horizontal del movimiento es amplificada por la multi reflexión de las ondas S al llegar a la superficie, mientras que la componente vertical es amplificada por la multi reflexión de las ondas P. Además, considera que las fuentes artificiales prevalecen mayoritariamente en la componente vertical del movimiento, las cuales tienden a inducir ondas Rayleigh que pueden ser cuantificadas determinando la razón entre la componente vertical del movimiento en superficie y en la base del depósito (Nakamura, 1989).

Es importante señalar que a pesar de que el método de Nakamura permitiría obtener la función de transferencia de un depósito de suelos en términos de frecuencia y amplificación, se ha comprobado empíricamente que su principal utilidad está en la determinación de la frecuencia fundamental en suelos blandos. Asimismo, provee una estimación gruesa del contraste de impedancias de los estratos en profundidad.

\section{Edificios severamente dañados en la Provincia de Santiago}

Se consideraron edificios, de tres o más pisos, que presentaron daños estructurales severos, siendo las estructuras categorizadas como habitables sólo después de refuerzo estructural y las estructuras irreparables con decretos de demolición. En ese contexto, de acuerdo al catastro de daños efectuado por Vergara (2015), se contabilizaron 68 edificios. Cabe destacar que en algunos casos las estructuras dañadas corresponden a blocks habitacionales pertenecientes a las mismas villas o condominios. En la Figura 1 se presenta la ubicación de los edificios considerados.

Se observa una concentración de edificios severamente dañados en la zona centro y norte de la Provincia de Santiago. En particular se presenta una mayor densidad de daños en las siguientes comunas:

- Quilicura: correspondiente a blocks de la Población Parinacota

- Maipú: edificios Don Luis Gandarillas, Tristán Valdés, Hermanos Carrera, entre otros. 
Figura 2: Daños observados en muros de hormigón armado de edificios, a) Huechuraba, daños en hall de entrada, b) Independencia, daño por flexo compresión y c) Macul, daño por flexo compresión

mediciones de vibraciones de 30 minutos de duración en las componentes Norte-Sur, Este-Oeste y Vertical, a una tasa de muestreo de $128 \mathrm{~Hz}$, incorporando las coordenadas geográficas de la medición. Se contabilizaron 142 mediciones, de las cuales 122 registros se ubicaron en las cercanías de los 68 edificios severamente dañados. Adicionalmente, se efectuaron 12 mediciones en las inmediaciones de las siguientes estaciones sismológicas: en el Centro de Referencia de Salud de Maipú, en el Campus Antumapu de la Universidad de Chile y en el Hospital Doctor Luis Tisné en Peñalolén. Asimismo, se efectuaron 8 mediciones en los alrededores del Aeropuerto de Pudahuel y en la comuna de Peñalolén. En la Figura 3 se presenta la ubicación de las mediciones efectuadas y las estaciones sismológicas.

Figura 1: Ubicación de edificios dañados en la Provincia de Santiago

De acuerdo a los análisis efectuados a los tipos de daños presentados en estructuras de hormigón armado durante el $27 \mathrm{~F}$, se han identificado patrones que se mantuvieron constante en distintas ciudades afectadas. Los daños corresponden a fallas por corte en vigas cortas y machones, falla por flexo-compresión en muros, fallas debido a irregularidad estructural, fallas de corte o flexión en conexiones viga-columna y viga-muro (Massone y Rojas, 2012). En la Figura 2 se presentan algunos ejemplos de daños observados en edificios de la Provincia de Santiago.

\section{Programa de mediciones vibraciones ambientales}

Se llevó a cabo un programa de mediciones de junio a septiembre 2016, utilizando dos equipos digitales Tromino ENGY de Micromed S.p.A (tromino.it). equipados con una antena GPS interna. En cada estación se registraron

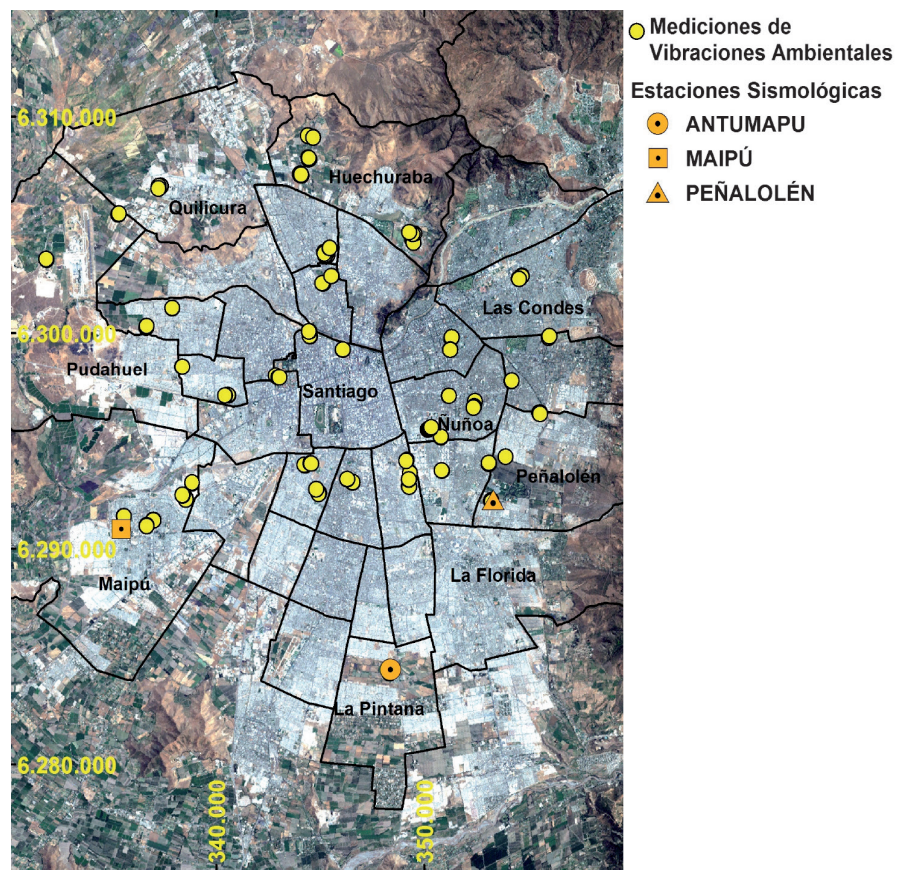

Figura 3: Ubicación mediciones de vibraciones ambientales y estaciones sismológicas 


\section{Evaluación de razones espectrales H/V}

Para cada medición de vibraciones ambientales se calculó su respectiva razón espectral mediante el software Geopsy. Esta herramienta se desarrolló en el proyecto europeo SESAME (2004), encontrándose disponible gratuitamente en internet en geopsy.org (Using ambient vibration techniques for site characterization, seismic microzonation and building). Para el cálculo de razones espectrales Geopsy utiliza un criterio de selección de partes de la señal, denominado ventanas, el cual permite identificar y eliminar los sectores donde existe un aumento abrupto de la señal, el cual estaría asociado a vibraciones artificiales. Para la selección de ventanas se utilizan los términos $S T A$ y LTA. Donde STA corresponde a la amplitud promedio de la señal en un periodo corto de tiempo (tsta) y LTA la amplitud promedio en un periodo largo de tiempo (tlta). Las ventanas se obtienen al comparar el cociente $S$ (donde $S=S T A / L T A)$ con valores umbrales $S_{\text {max }}$ y $S_{\text {min }}$ definidos anteriormente.

En consecuencia, definiendo un valor de tlta fijo igual al largo de la ventana $L_{w}$ se obtiene $L T A$, luego mediante una ventana móvil de largo tsta, la cual recorre todo el intervalo tlta, se va calculando instantáneamente STA. Si el cociente $S$ se enmarca dentro de los valores umbrales, se considera que la ventana contiene vibraciones permanentes $y$, por lo tanto, es utilizada para el cálculo de las razones espectrales. La Tabla 1 presenta los valores utilizados para el criterio de selección de ventanas y la Figura 4 muestra un ejemplo de ventanas seleccionadas en Geopsy. Adicionalmente, se utilizó para el suavizado de señales la función definida por Konno y Ohmachi (1998) con un coeficiente de ancho de banda $b$ igual a 40. Finalmente, la razón espectral se obtiene como el promedio del cociente entre el espectro de la componente horizontal combinada y el espectro de la componente vertical en cada ventana seleccionada.

Tabla 1: Valores utilizados para el criterio de selección de ventanas

\begin{tabular}{|c|c|}
\hline Símbolo & Valor \\
\hline$L_{\mathrm{w}}$ & $25 \mathrm{~s}$ \\
\hline tlta & $25 \mathrm{~s}$ \\
\hline$t_{\text {sta }}$ & $1 \mathrm{~s}$ \\
\hline$S_{\text {máx }}$ & 0.5 \\
\hline$S_{\text {min }}$ & 2.0 \\
\hline
\end{tabular}

\section{Resultados obtenidos}

A partir de los resultados obtenidos de las mediciones se definieron 4 tipos de razones espectrales los cuales se grafican en la Figura 5 y se definen a continuación:

- Tipo A: Razones Espectrales con peak muy marcado y amplitudes mayores o iguales a 3.

- Tipo B: Razones Espectrales con peak marcado y amplitudes mayores o iguales a 2 , pero menores a 3 .

- Tipo C: Razones Espectrales con peak poco claro y amplitudes mayores o iguales a 2, pero menores a 3 .

- Tipo D: Razones Espectrales planas con amplitudes menores a 2 o un peak con frecuencias mayores a 5 $\mathrm{Hz}$.

Definiciones de tipos de razones espectrales H/V similares han sido propuestas anteriormente (Pastén, 2007). Es importante señalar que los tipos A y B están asociados a depósitos de suelos no rígidos. En cambio, los tipos $\mathrm{C}$ y D corresponden a resultados característicos de depósitos rígidos.

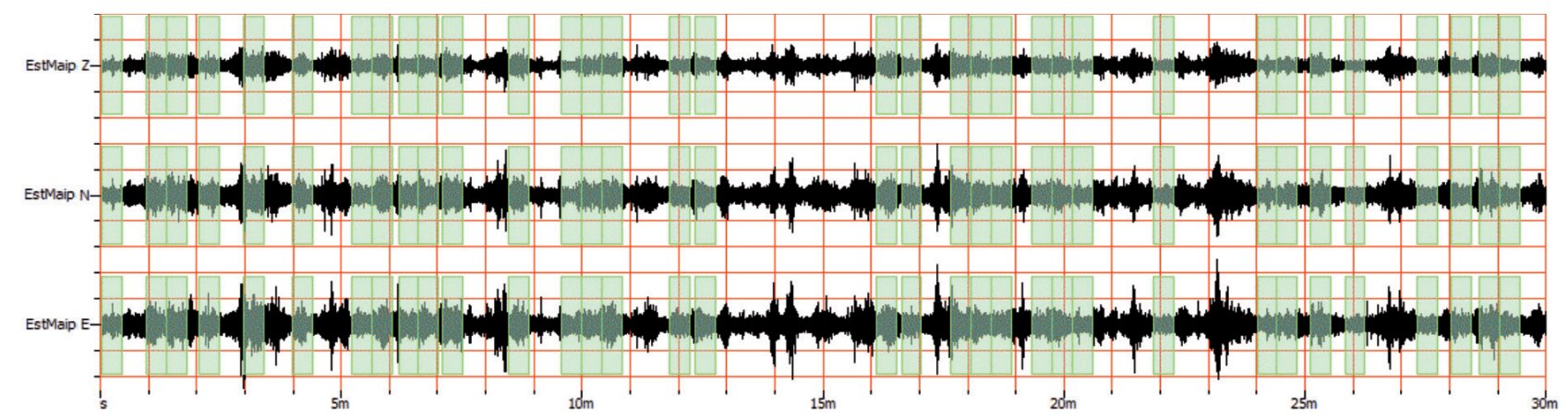

Figura 4: Ejemplo de ventanas utilizadas según criterio de selección 

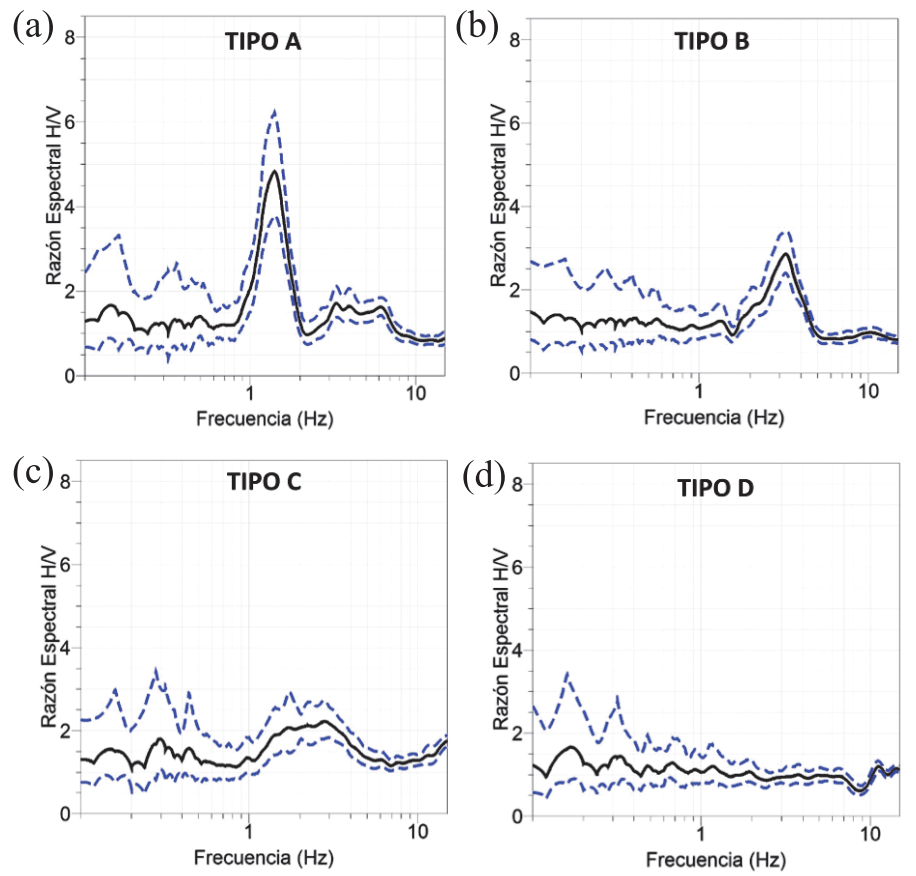

Figura 5: Ejemplos de tipos de razón espectral en las comunas de: a) Huechuraba, b) Maipú, c) Cerro Navia y d) Las Condes

En el marco anterior, en la Figura 6a se presenta un mapa donde se muestra la geología de la cuenca de Santiago y la profundidad del basamento rocoso. La morfología del basamento rocoso corresponde al modelo gravimétrico tridimensional desarrollado por Araneda et al. (2000). Por otro lado, el mapa geológico corresponde a las 10 unidades geológicas definidas por Leyton et al. (2010), las cuales fueron obtenidas utilizando información de pozos y de los estudios desarrollados por Valenzuela (1978), Wall et al. (1999), Milovic (2000), Fernández (2001, 2003), Sellés y Gana (2001), Rauld (2002) y Fock (2005). En la Tabla 2 se definen las unidades geológicas. En la Figura $6 \mathrm{~b}$ se presentan los resultados de las mediciones de acuerdo al tipo de razón espectral obtenido.

\section{Análisis de resultados}

Considerando los resultados de razones espectrales, se desarrolló un mapa de zonificación de los tipos de suelos (ver Figura 6b), detallado a continuación:

- Suelos no rígidos: Corresponde a la unidad VII (suelo fino) donde se obtuvo periodos mayores a $0.5 \mathrm{~s} \mathrm{y}$ razones espectrales Tipo A.

- Suelos semi rígidos: Engloban sectores de la unidad VII y la ceniza volcánica VI con periodos que varían entre 0.2 a $0.5 \mathrm{~s}$ y razones espectrales Tipo A y B.

- Suelos rígidos: Corresponden a las unidades geológicas II, IIIa y VIII con periodos menores a $0.2 \mathrm{~s}$ y razones espectrales Tipo D y en algunos casos Tipo C.

Analizando la relación entre las características geotécnicas
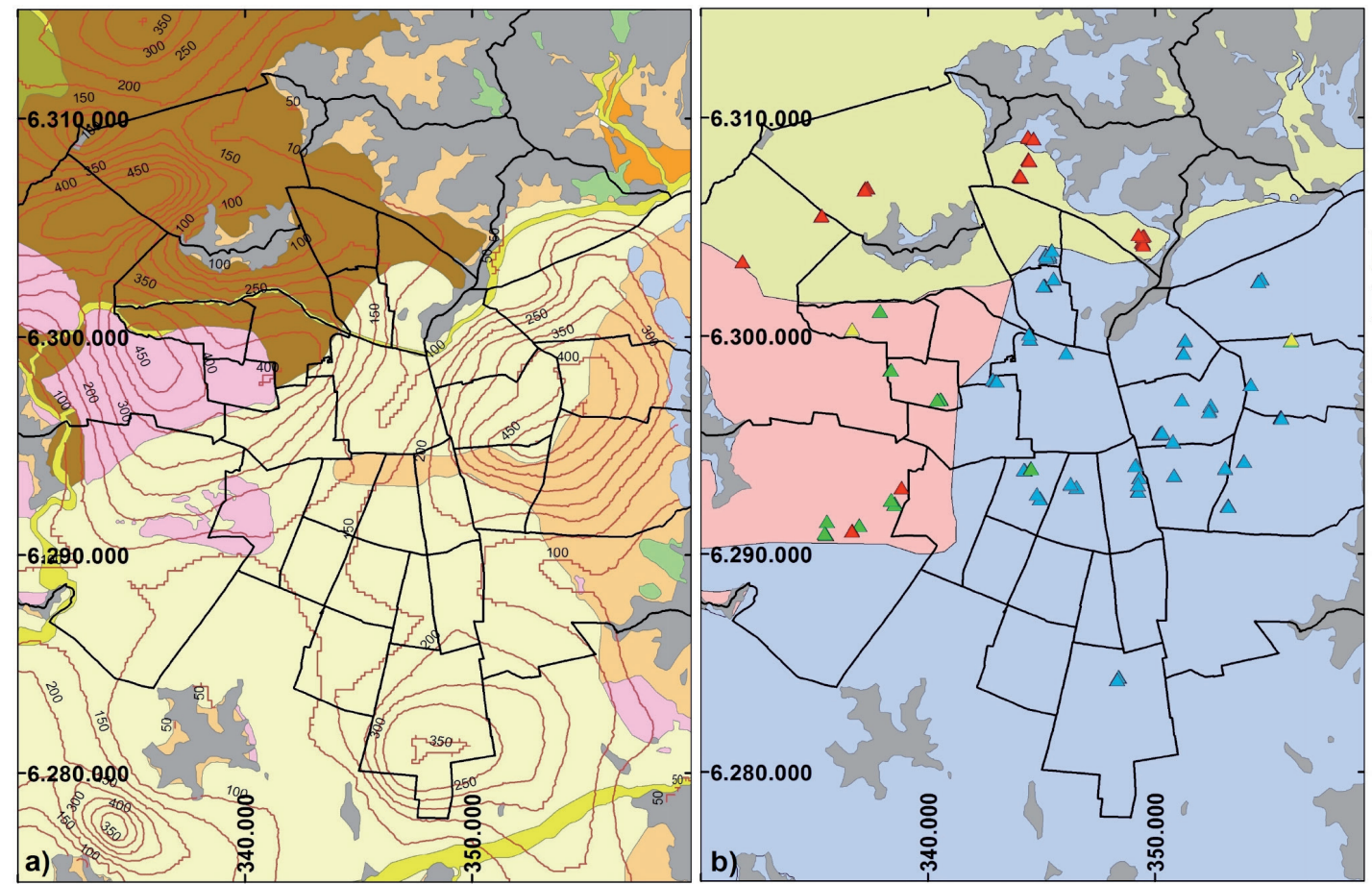

Tipo Razón Espectral

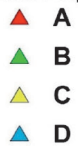

$\triangle B$

$\triangle \mathbf{C}$

$\triangle \mathrm{D}$

Tipo de Suelos

No rígidos, $T>0.5 \mathrm{seg}$

Semi -rígidos, $0.2<T \leqslant 0.5 \mathrm{seg}$.

Rígidos,

registros planos o $T \leqslant 0.2 \mathrm{seg}$.

- Profundidad Roca

Unidades Geológicas

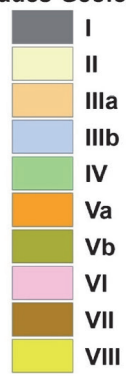

Figura 6: a) Geología superficial y profundidad del basamento rocoso y b) zonificación de tipo de suelos según Razones Espectrales $\mathrm{H} / \mathrm{V}$ 
Tabla 2: Unidades geológicas presentes en la cuenca de Santiago

\begin{tabular}{|c|l|}
\hline Unidad & Descripción \\
\hline I & $\begin{array}{l}\text { Roca. Aflora en las cadenas montañosas alrededor de } \\
\text { la cuenca y en cerros aislados }\end{array}$ \\
\hline II & $\begin{array}{l}\text { Gravas fluviales. Material grueso, principalmente } \\
\text { gravas arenosas }\end{array}$ \\
\hline IIIa & $\begin{array}{l}\text { Depósitos aluviales. Bloques y gravas en una matriz } \\
\text { areno arcillosa con intercalaciones de arena, limo y } \\
\text { arcillas }\end{array}$ \\
\hline IIIb & $\begin{array}{l}\text { Depósitos aluviales antiguos. Gravas y bloques en } \\
\text { matriz areno arcillosa. Corresponde a viejos abanicos } \\
\text { aluviales }\end{array}$ \\
\hline IV & $\begin{array}{l}\text { Gravas y bloques en una matriz arcillo arenosa } \\
\text { asociada a un antiguo deslizamiento }\end{array}$ \\
\hline Va & $\begin{array}{l}\text { Depósitos aluviales. Gravas en una matriz arcillo } \\
\text { arenosa con intercalaciones de arena, limo y arcilla. } \\
\text { Además de una gruesa capa de suelo arcillo limoso } \\
\text { asociado a esteros Colina y Arrayán }\end{array}$ \\
\hline Vb & $\begin{array}{l}\text { Depósitos aluviales. Arenas finas a gruesas mezcladas } \\
\text { con limos y arcillas asociado al estero Lampa }\end{array}$ \\
\hline VI & $\begin{array}{l}\text { Depósito de ceniza volcánica de más de 20 m de } \\
\text { espesor con intercalaciones de arenas, limos y gravas. } \\
\text { Denominado Ignimbrita de Pudahuel }\end{array}$ \\
\hline VII & $\begin{array}{l}\text { Depósito de suelos finos. Limos y arcillas con } \\
\text { intercalaciones de gravas, arenas y cenizas volcánicas }\end{array}$ \\
\hline VIII & $\begin{array}{l}\text { Depósitos fluviales recientes. Granulometría variable } \\
\text { desde gravas a limos }\end{array}$ \\
\hline
\end{tabular}

y geomorfológicas de la cuenca de Santiago con las razones espectrales se observa que sobre la unidad VII (suelos finos), se presentaron los periodos más altos (mayores a $0.5 \mathrm{~s}$ ), al acercarse a los límites donde esta unidad interdigita con sedimentos más competentes, los periodos disminuyen, como es el caso de la zona noroeste de Huechuraba donde interdigitan las unidades VII y IIIa, y en la comuna de Cerro Navia donde interdigitan las unidades VI y VII.

Por otra parte, consistentemente sobre la Ignimbrita de Pudahuel (unidad VI), se presentan periodos entre 0.2 a 0.5 $\mathrm{s}$, siendo estas mediciones efectuadas en distintas comunas como Pudahuel, Maipú, Cerro Navia y Lo Prado.

Con respecto al tipo de Razón Espectral, se observa que las razones espectrales donde es posible identificar un peak de frecuencia (Tipo A y B) se emplazan sobre suelos finos (unidad VII) y ceniza volcánica (VI). En el caso de la unidad IIIa, caracterizada por un contenido variable desde gravas a material más fino, se observa principalmente razones espectrales Tipo D, correspondiente a suelos rígidos. No obstante, se presentaron particularmente en la parte distal de la unidad una razón espectral Tipo B en Pedro Aguirre Cerda y Tipo C en Las Condes. Lo anterior podría deberse a que en la parte distal de un abanico aluvial se encuentran depósitos de sedimentos finos con un alto contenido arcilloso, en zonas de baja pendiente donde se pueden asociar a flujos de menor energía (Fernández, 2003). En consecuencia, en la parte distal de la unidad IIIa habría sedimentos más finos, los que se evidenciarían con razones espectrales con peaks claros, sin embargo, al estar próximos a suelos más rígidos presentan periodos bajos.

En el caso de la unidad II, consistentemente se identificaron razones espectrales Tipo D, característicos de suelos rígidos. Sin embargo, en las comunas de Independencia y Conchalí se identificaron razones espectrales planas (Tipo D) sobre suelos finos (unidad VII), lo cual podría deberse a que el sector donde entran en contacto las unidades II y VII sería más hacia el norte, es decir, las zonas donde se midieron las razones tipo D estarían emplazados sobre suelos más rígidos. En base a esto, la zonificación incluyó dichos puntos como suelos rígidos.

Con respecto a la profundidad del basamento rocoso, para las mediciones H/V Tipo D (suelo rígido) no se observan variaciones de éstas con la profundidad de la roca. En este trabajo no se tienen mediciones de razones espectrales H/V del Tipo A, B y C con variación de la profundidad de la roca.

Por otra parte, al comparar los resultados obtenidos en la Figura 6 con la ubicación de los edificios dañados mostrados en la Figura 1, se observa que no existe un periodo fundamental preponderante en las cercanías de los edificios dañados, ya que éstos presentan un rango variable de periodos. Por ejemplo, en la cuidad empresarial en Huechuraba (edificios Patio Mayor 937, 945 y 961), se registraron periodos mayores a $0.5 \mathrm{~s}$ y en Maipú en las cercanías de los edificios Condominio Hermanos Carrera, Tristán Valdés y Luis Gandarillas, se presentaron periodos menores, entre 0.2 a $0.5 \mathrm{~s}$. Siendo en ambos casos edificios de 4 y 5 pisos.

Asimismo, los edificios severamente dañados se ubican sobre distintos tipos de razones espectrales, desde Tipo A característicos de suelos blandos hasta razones espectrales Tipo D característicos de suelos rígidos. 


\section{Conclusiones}

Con el objetivo de identificar si el fenómeno de amplificación sísmica fue un factor influyente en los edificios severamente dañados durante el 27F, se realizaron mediciones de vibraciones ambientales en el entorno de los 68 edificios más dañados en la Provincia de Santiago. De acuerdo a los resultados entregados por 142 mediciones de razones espectrales, se concluye que no existe correlación evidente entre las características de la razón espectral y los edificios severamente dañados, debido a que no se observa un patrón determinado de periodos o tipo de razón espectral del terreno de fundación de los edificios.

Por ejemplo, existen edificios emplazados sobre suelos rígidos, cuyos daños resultan difícil de explicar por la alta competencia de estos suelos. En este sentido, si el suelo es rígido no existe una frecuencia en particular que amplifique el suelo, ya que todos los niveles de amplificación son semejantes y además, bajos, de esta manera el efecto de sitio no afecta de una forma particular a las estructuras que están emplazadas en este tipo de suelo, con lo cual es posible señalar que los daños en estos casos se deben a alguna deficiencia estructural. Por el contrario, en el caso de los edificios ubicados en suelos no rígidos y semirígidos, los daños estructurales podrían eventualmente ser atribuidos al fenómeno de efecto de sitio.

Análogamente, en base a los resultados obtenidos se categorizaron los tipos de suelo de la cuenca de Santiago en tres grupos: suelos no rígidos con periodos mayores a $0.5 \mathrm{~s}$, suelos semi-rígidos de periodos entre 0.2 a $0.5 \mathrm{~s}$ y suelos rígidos asociados a registros planos o periodos menores a $0.2 \mathrm{~s}$. Esta categorización se considera útil para el análisis estructural de nuevas obras, ya que las estructuras construidas en suelos no rígidos o semi-rígidos podrían estar afectados por la amplificación sísmica.

Por otra parte, se corrobora la correlación entre las características geotécnicas del suelo y el tipo de razón espectral, ya que en suelos blandos (Unidad VII) y ceniza volcánica (VI) se identificaron razones espectrales con peaks claros y periodos fundamentales mayores a $0.5 \mathrm{~s}$ (unidad VII) y entre 0.2 a $0.5 \mathrm{~s}$ (unidad VI). Asimismo, en el caso de suelos rígidos (unidad II) se identifican razones espectrales planas. En consecuencia, el método de Nakamura permite identificar periodos fundamentales de suelos blandos.
Por otra parte, para suelos rígidos no se observa correlación entre las mediciones $\mathrm{H} / \mathrm{V}$ (Tipo D) con la profundidad del basamento rocoso. Además, el daño estructural observado se produce tanto en sectores más profundos del basamento rocoso, por ejemplo Ñuñoa, como en sectores menos profundos, por ejemplo Independencia.

\section{Referencias}

Araneda, M., Avendaño, M. y Merlo, C. (2000). Modelo gravimétrico de la cuenca de Santiago, etapa III final. IX Congreso Geológico de Chile, Puerto Varas, Chile, 2, 404-408

Fernández, J.C. (2003). Respuesta sísmica de la cuenca de Santiago. Servicio Nacional de Geología y Minería. Carta Geológica de Chile. Serie Geología Ambiental N 1

Fernández, J.C. (2001). Estudio geológico-ambiental para la planificación territorial del sector Tiltil - Santiago. Memoria de título de Geólogo, Universidad de Chile

Fock, A. (2005). Cronología y tectónica de la exhumación en el Neógeno de Los Andes de Chile Central entre los $33^{\circ}$ y los $34^{\circ}$ $S$. Tesis de Magíster, Universidad de Chile

Konno, K. and Ohmachi, T. (1998). Ground-motion characteristics estimated from spectral ratio between horizontal and vertical components of microtremor. Bulletin of the Seismological Society of America 88(1), 228-241

Leyton, F., Sepúlveda, S., Astroza, M., Rebolledo, S., González, L., Ruiz, R., Foncea, C., Herrera, M. y Lavado, J. (2010). Zonificación sísmica de la cuenca de Santiago. X Congreso Chileno de Sismología e Ingeniería Antisísmica. ACHISINA, Santiago

Massone, L. y Rojas, F. (2012). Comportamiento de edificios de hormigón armado. En $M w=8.8$ Terremoto en Chile. 27 de febrero 2010. Universidad de Chile

Milovic, J.J. (2000). Estudio geológico-ambiental para el ordenamiento territorial de la mitad sur de la cuenca de Santiago. Memoria de título, Universidad de Concepción

Nakamura, Y. (2008). On the H/V spectrum. The $14^{\text {th }}$ World Conference on Earthquake Engineering, Beijing, China

Nakamura, Y. (1989). A method for dynamic characteristics estimation of subsurface using microtremor on the ground surface. Quarterly Reports of the Railway Technical Research Institute 30(1), 25-33 
Pastén, C. (2007). Respuesta sísmica de la cuenca de Santiago. Tesis de Magíster, Universidad de Chile

Presidencia de la República (2013). La reconstrucción de Chile. Colección Memoria Presidencial. Santiago, Chile

Rauld, R. (2002). Análisis morfoestructural del frente cordillerano Santiago Oriente, entre el río Mapocho y quebrada de Macul. Memoria de título de Geólogo, Universidad de Chile

Sellés, D. y Gana, P. (2001). Geología del área de Talagante-San Francisco de Mostazal, regiones Metropolitana de Santiago y del Libertador General Bernardo O'Higgins. Servicio Nacional de Geología y Minería. Carta Geológica de Chile. Serie Geológica Básica, $\mathrm{N}^{\circ} 74$

SESAME (2004). Site effects assesment using ambient excitations. Report on Simulation for real sites. European Commission - Research General Directorate

Valenzuela, G. (1978). Suelo de fundación de Santiago. Instituto de Investigaciones Geológicas. Boletín N`33
Vergara, L. (2015). Relación entre las propiedades geológicas y geotécnicas de la cuenca de Santiago y los daños observados en el terremoto del 27 de Febrero del 2010. Tesis de Magíster, Universidad de Chile

Vergara, L. y Verdugo, R. (2014). Condiciones geológicasgeotécnicas de la cuenca de Santiago y distribución de daños del terremoto del 27F. VIII Congreso Chileno de Ingeniería Geotécnica, SOCHIGE, Santiago

Wall, R., Sellés, D. y Gana, P. (1999). Área Tiltil-Santiago, Región Metropolitana. Servicio Nacional de Geología y Minería. Mapas Geológicos N¹1 